D. Crocker (UCLA-NMC)

RFC 657, NIC 31160 (Oct. 25, 1974)

Online file: [ISI] $<$ DCROCKER>NAOVTD.TXT

\title{
TELNET OUTPUT VERTICAL TAB DISPOSITION OPTION
}

1. Command name and code

NAOVTD 15

(Negotiate About Output Vertcial Tab Disposition)

2. Command meanings

In the following, we are discussing a simplex connection, as described in the NAOL and NAOP Telnet Options specifications.

IAC DO NAOVTD

The data sender requests or agrees to negotiate about output vertical tab character disposition with the data receiver. In the case where agreement has been reached and in the absence of further subnegotiations, the data receiver is assumed to be handling output vertical tab character considerations.

IAC DON'T NAOVTD

The data sender refuses to negotiate about output vertical tab character disposition with the data receiver, or demands a return to the unnegotiated default mode.

IAC WILL NAOVTD

The data receiver requests or agrees to negotiate about output vertical tab character disposition with the sender. In the case where agreement has been reached and in the absence of further subnegotiations, the data receiver alone is assumed to be handling output vertical tab character considerations.

IAC WON'T NAOVTD

The data receiver refuses to negotiate about output vertical tab character disposition, or demands a return to the unnegotiated default mode.

IAC SB NAOVTD DS <8-bit value> IAC SE

The data sender specifies, with the 8-bit value, which party should handle output vertical tab characters and what their disposition should be. The code for DS is 1.

IAC SB NAOVTD DR <8-bit value> IAC SE

The data receiver specifies, with the 8-bit value, which party should handle output vertical tab characters and what their disposition should be. The code for DR is 0 .

3. Default

DON'T NAOVTD/WON'T NAOVTD

In the default absence of negotiations concerning which party, data sender or data receiver, is handling output vertical tab character considerations, neither party is required to handle vertical tab characters and neither party is prohibited from handling them; but it is appropriate if at least the data receiver handles vertical tab character considerations, albeit primitively.

4. Motivation for the Option

Please refer to section 4 of the NAOL and of the NAOVTD Telnet option descriptions. 
5. Description of the Option

The data sender and the data receiver use the 8-bit value along with the DS and DR SB commands as follows:

8 bit value Meaning

0

1 to 250

251

252

253

254

255
Command sender suggests that he alone will handle vertical tab characters, for the connection. Command sender suggests that the other party alone should handle tab characters, but suggests that a delay of the indicated value be used. The value is the number of character-times to wait or number of NULs to insert in the data stream before sending the next data character.

Command sender suggests that the other party alone handle vertical tabs, but suggests that each occurrence of the character be replaced by carriage-return/linefeed.

Command sender suggests that the other party alone handle vertical tabs, but suggests that they be discarded. Command sender suggests that the other party alone should handle tab characters, but suggests that tabbing be simulated.

Command sender suggests that the other party alone should handle the output disposition but suggests waiting for a character to be transmitted (on the other simplex connection) before sending more data. Note that, due to the assynchrony of the two simplex connections, phase problems can occur with this option.

Command sender suggests that the other party alone should handle the output disposition and suggests nothing about how it should be done.

The guiding rules are that:

1. if neither data receiver nor data sender wants to handle the output vertical tab characters, the data receiver must do it, and 2. if both data receiver and data sender want to handle the output vertical tab characters, the data sender gets to do it.

The reasoning for the former rule is that if neither want to do it, then the default in the NAOVTD option dominates. If both want to do it, the sender, who is presumed to have special knowledge about the data, should be allowed to do it, taking into account any suggestions the receiver may make. Simulation is defined as the replacement of the character by enough line-feeds (only) to advance the paper (or line-pointer) to the next vertical tab stop.

Note that delays, controlled by the data sender, must consist of NUL characters, inserted immediately after the line-feed character. This is necessary due to the assynchrony of network transmissions. As with all option negotiations, neither party should suggest a state already in effect except to refuse to negotiate; changes should be acknowledged; and once refused, an option should not be resuggested until "something changes" (e.g., another process starts). At any time, either party can disable further negotiation by giving the appropriate WON'T NAOVTD or DON'T NAOVTD command. 